\title{
Webots-based Simulator for Biped Navigation in Human-living Environments
}

\author{
Zeyang Xia†, Xiaojun Wang $\dagger$, Yangzhou Gan, Thomas-Glyn Hunter Cox, \\ Xue Zhang, Huang Li, Jing Xiong*
}

\begin{abstract}
Navigation is one of the key issues of biped robot, especially in complicated and uncertain human-living environment. There have been challenges for ensuring the stability, efficiency and security of the biped navigation system. In this paper, a framework utilizing sampling-based footstep planner is proposed for the simulation of the biped navigation. Sensor fusion method is adopted to process and generate the correlated environment information for footstep planning. Two specific experiments have been conducted to validate the functionality and performance of the proposed framework.
\end{abstract}

\section{INTRODUCTION}

Recently with the Fukushima nuclear accident and the robot competition (DARPA Robot Challenge), biped robots have shown great advantages compared to wheeled robots in task-oriented situations. Biped robots have been regarded as the execution unit of handling the dangerous tasks as well as the service agent in the future. Biped robots are able to adapt to complex environment and deal with difficult tasks. Biped navigation is one of the essential functionalities for the biped robots to accomplish high-level tasks.

Due to the complex structure and the safety consideration of robots, it may be difficult to carry out physical experiments to validate the testing algorithm and program. Thus, the usage of a simulator is often of significant importance. As a tool to test the efficiency, safety, and robustness of new algorithm, the simulator can greatly reduce the experimental time and cost. For biped navigation, because of the capability to step over and step upon, the boundary between obstacles and the environment has been blurred such that conventional path planning is not suitable [1]. Kuffner et al. [2]-[4] proposed a sampling-based approach for global footstep planning. Upon the sampling-based approach, Chestnutt et al. proposed to use $A^{*}$ search to compute foot sequence by applying the dynamically adjustable action model [5] and adaptive action model to perform the local searches based on reference actions [6]. Xia et al. proposed a randomized sampling-based footstep planner based on rapidly exploring random trees (RRT) algorithm to resolve the footstep planning problem in

This research was supported by National Science Foundation of China (No. 51305436, 61403368).

Z. Xia, X. Wang, Y. Gan, X. Zhang and H. Li are with Shenzhen Institutes of Advanced Technology, Chinese Academy of Sciences, and The Chinese University of Hong Kong, Shenzhen, China. J. Xiong is with Shenzhen Institutes of Advanced Technology, Chinese Academy of Sciences, Shenzhen, China. T. H. Cox is with Purdue School of Engineering and Technology, Indiana University Purdue University Indianapolis, U.S.

$\dagger$ These authors contributed equally to this work.

*Author to whom correspondence should be addressed. Email: jing.xiongesiat.ac.cn; Phone: +86-755-86392298 the environments with local loops or narrow passages [7]-[9]. Theses researches mentioned above have proved the great advantage of the $A^{*}$ sampling-based footstep planner.

Conventional locomotion requires a sophisticated control method including an accurate robot modeling and complex dynamic calculation based on the stability control to keep the robot functional. Nowadays more and more researchers have studied the control methodology inspired from animal locomotion which has evolved to be efficient, rapid and reliable [10]. CPG controller, one of the bionic based controllers, consists of layered networks capable of producing coordinated oscillatory patterns of rhythmic activity which receiving adjustment signals from higher control centers [11].

This paper proposes a generalized framework for the simulation of biped navigation in human-living environment. The A* sampling-based footstep planner was utilized as the searching engine to calculated the proper footstep sequence. A collision free element step library based on the model of physical robot was built as the database for the footstep planner. The footstep planning and execution adopt the "Offline planning, online calling" to reduce the planning cost and execution speed. The execution of the element step was conducted using an open-loop control methods since based on the experiment data analysis, the well-calculated joint values would minimized the error caused by slipping. The element step library, including the footstep placements of the robot and the correlated footstep-gesture transition trajectory, was calculated based on the stability control of the Center of Mass (CoM).

The rest of this paper is organized as follows. Section II introduces the system architecture and the procedure of the simulation experiment. Section III illustrates the detailed functions used in the biped navigation. Section IV presents the experimental results. Section V concludes this paper.

\section{FRAMEWORK}

The framework for simulation of biped navigation is composed of two parts. The first part is the construction of the Element Step Library, which is the database for the footstep planner and the execution element for the offline planning. The element steps not only consider the footstep?s placement but also the corresponding transition posture. The second part is the environment sensing which generate the real-time map and the goal position for the footstep planner to update the footstep sequence. Figure 1 shows the diagram of the biped walking. 


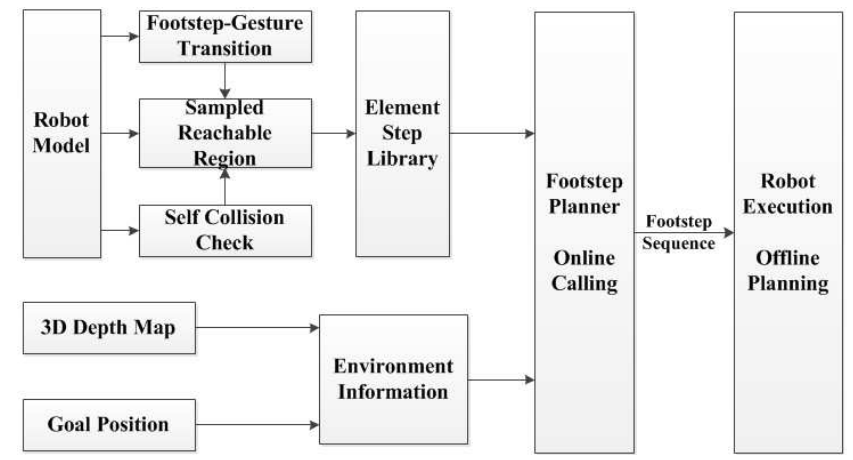

Fig. 1. The flow diagram for the biped walking

\section{A. Construction of Element Step Library}

1) Sampled Reachable Region: Sampling-based footstep planner starts from estimating the entire reachable region to find the available footstep placements. By examining the physical limitation of the robot?s forward, sidewards and rotation movement, a library containing all the basic footstep placements is built.

2) Self-Collision Check: Elements in the element step library, such as the inward slide and the forward-inward rotation footstep placement, may cause self-collision. To avoid the self-collision, some elements is removed from the library based on the Kinematic analysis and experiment test.

3) Footstep-Gesture Transition: Footstep-Gesture Transition mainly considers the stability of the robot when the footstep placements change from one foot to the other foot. This procedure consists of the one foot support phase and the movement of the CoM during two feet support phase. Detailed procedure is illustrated in Section III.

\section{B. Environment Sensing}

1) 3D Depth Map: The environmental information updating is of great importance for biped navigation in humanliving environment. The synchronization between the execution of the element step and the speed of the map generation should be considered to avoid the robot from colliding obstacles. The noise caused by the robot?s movement is filtered to avoid the searching failure. Detailed depth map generation algorithm is presented in Section III.

2) Goal Position: The goal position of the biped navigation is predefined by user. The goal needs to be distinctive from the surrounding environment such that color or shape based segmentation is sufficient to extract the goal position.

\section{KEY FUNCTIONS}

To realize the biped navigation, the construction of the Element Step Library and the generation of the 3D Depth Map are necessary. The proper element step library guarantees the stability of the walking. The accurate $3 \mathrm{D}$ depth map guides the robot to avoid the obstacles in the environment.

\section{A. Footstep-Gesture Transition}

1) Realization of the element steps: Fig. 2 shows the generalized sampled reachable region. To execute the element step, the robot needs to place the swing foot to the desired footstep placement. However, for continuous walking process, the swing and support foot is always changing between left and right, and the control of the CoM for the stability is required.

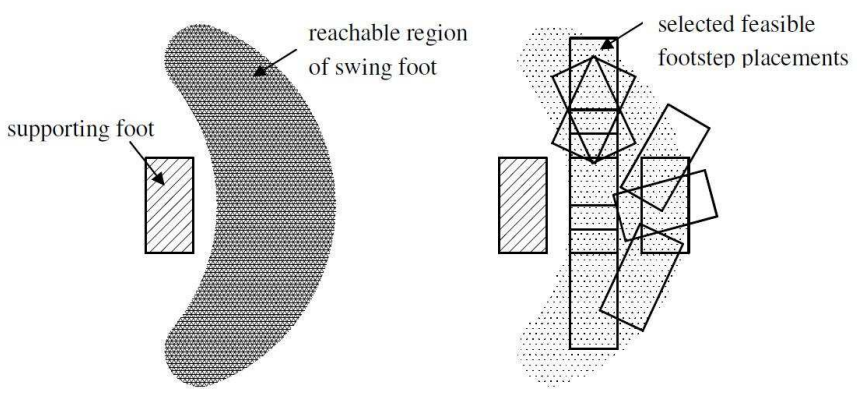

Fig. 2. Footstep-transition model. (Left) The reachable region for the swing foot, (Right) Sets of footstep placement defined in the transition model.[9]

Fig. 3 shows the theoretical trajectory of biped robot?s CoM during walking. It indicates that the walking process can be divided into the single foot support phase and double feet support phase. During the single foot support phase, the CoM falls into the projection region of the support foot, and as the robot?s swing foot moves from one footstep placement to a new footstep placement. During the double feet support phase, the CoM moves the previous support foot to the present support foot by adjusting the hip-roll and the ankleroll joints.

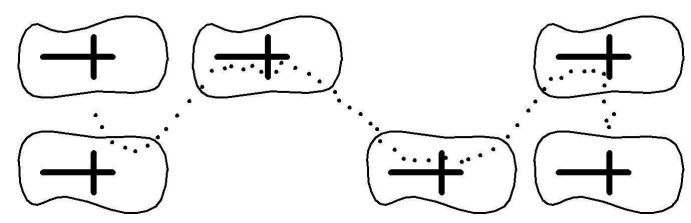

Fig. 3. Theoretical trajectory of biped robot?s CoM while walking

Fig. 4 shows the decomposing of one foot support phase into five key postures. The five key postures are used as the middle points to simplify the joints value calculation process. Element steps for each foot are connected by the middle "center posture" which is used as the starting and final posture (symmetric one) for each element step.

After space domain and timing domain adjustment, one element step is built starting from the center posture to a new center posture. Within the starting center posture, the CoM falls in the left or right foot as the present support foot, and within the new center posture, the CoM falls in the 


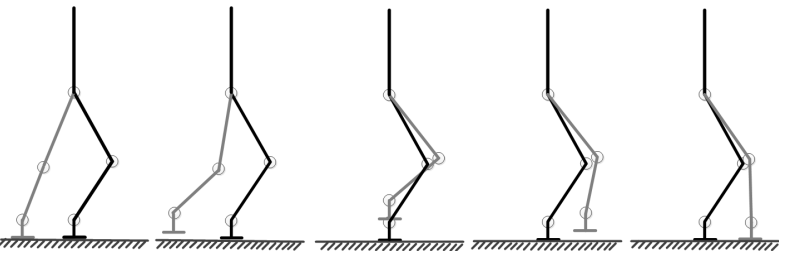

Fig. 4. Five key postures of the one foot support phase from present footstep placement to new footstep placement, they are the leaving ground posture, post leaving ground posture, center posture, pre-swing ground posture, swing ground posture, respectively. (Black lines indicate the support leg and foot while the gray ones show the movement of the swing leg and foot.)

right or left foot as the new support foot, which is placed in the desired footstep placement.

2) Re-examine element steps: During the experiment, certain sampled element steps, which are mainly near the extreme limitation of the forward and slide range, may be unstable even though parameters have been well adjusted. For safety consideration, these steps will be removed from the element step library.

3) Discussion: The construction of the element step library is the fundamental part of the sampling based searching algorithm: the further calculated step sequence is based the accurate position of the current step, therefore, errors within each element step would affect the final performance greatly. On the other hand, since the continuous movement has been divided into element steps, the calculation load is greatly reduced and the further fault isolation would be easier to conduct.

\section{B. $3 D$ Depth Map Generation}

The 3D Depth Map generation consists of two steps: the general environment sensing and the robot-The 3D Depth Map generation consists of two steps: the general environment sensing and the robot-related noise filtering.

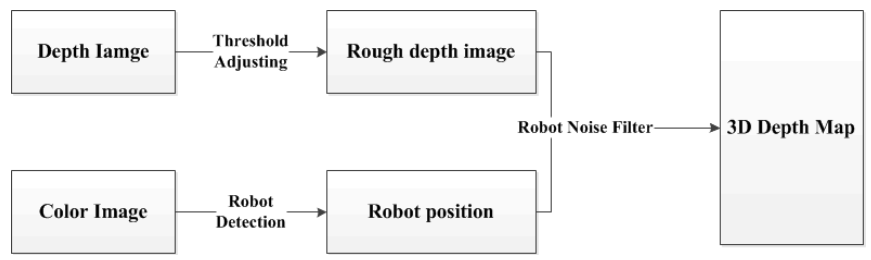

Fig. 5. The diagram of depth map generation.

1) Environment sensing to Depth map: For environment sensing, depth and color sensors fusion is applied to get the depth map of the environment. As the global environment information is needed for the sampling-based footstep planner, sensors with wide sensing region are required to build the depth map.

After acquiring the depth and color image, the valid experiment area (i.e. the region of interest) is first extracted using the color segmentation. Threshold adjustment on the depth image is then used to determine boundaries of obstacles. Multiple filters are used to remove spark noise.

2) Remove discrete noise caused by the robot: The procedure explained above is able to generate a proper 3D depth map for sampling-based footstep planner in static environment. While in human-living environment which is dynamic, the movement of the robot would influence the depth data and generate obstacle-like noise near the robot, thus lead to the failure of searching.

To remove the noise caused by the movement of the robot, we used the color based segmentation to isolate the robot from the environment, and then adopted the connectcomponent analysis methods to process the depth data to remove the "robot-related" noise near the robot. Generally, after these two steps, the 3D Depth Map for the footstep planner is sufficient to calculate the footstep sequence.

\section{EXPERIMENTS AND RESULTS}

Webots [12] is adopted as the simulation platform for experimental environment construction and the algorithm testing. The biped robot model is generated from an Aldebaran NAO robot with 21 degrees of freedom and a virtual Kinect sensor is used to sensing the environment. Fig. 6 shows the simulation scene.
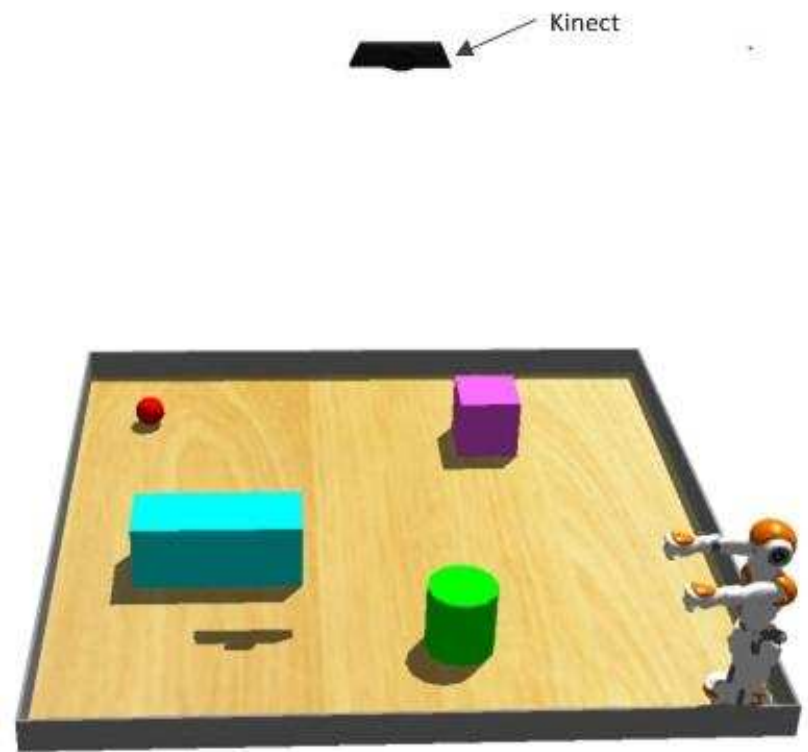

Fig. 6. The simulation scene. Kinect Sensor is installed onto the cailing and the goal position is represented by a moveable red ball. The experiment area has a size of $2 m^{*} 2 \mathrm{~m}$.

In the simulation experiments, a Kinect sensor [13] is installed onto the transparent ceiling about $2.7 \mathrm{~m}$ height from the floor. Fig. 7 shows the raw image acquired from the Kinect. 

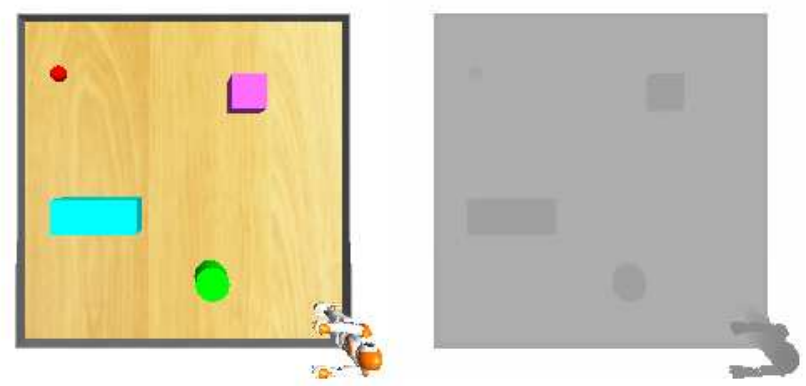

Fig. 7. Raw image from virtual Kinect

In our experiments, a 3D depth map with a region of pixels of $200^{*} 200$ is generated for the footstep planner.

\section{A. Element Step Library}

An Element Step Library containing 5 element steps (Fig. 8) is built as the database for the footstep planner.Specifications of the element step will be explained.
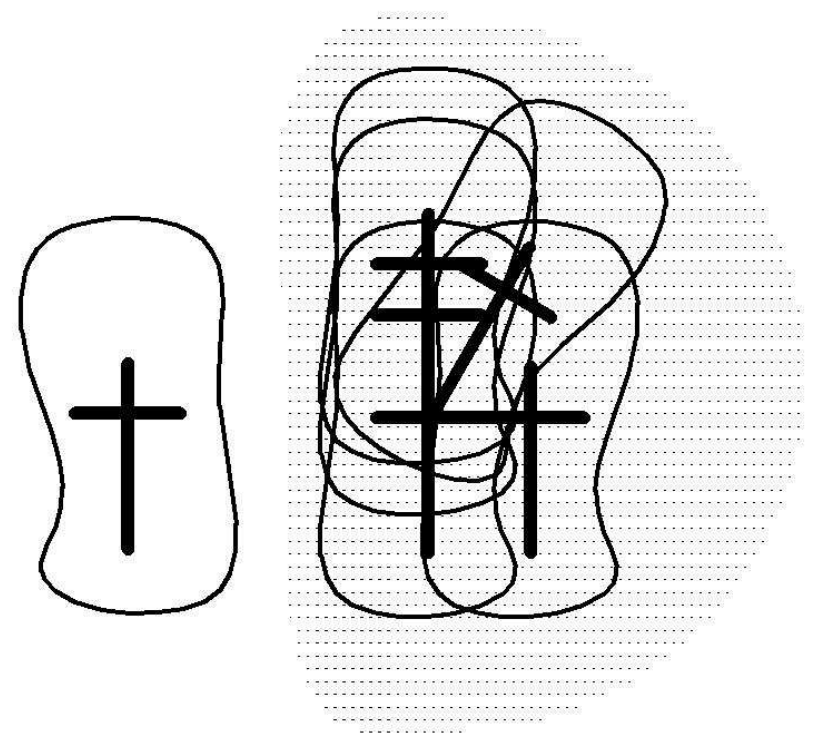

Fig. 8. Footsep placement for swing foot. Left rectangle indicates the support foot and the right rectangles indicates the avaiable footstep placement for the swing foot

TABLE I

ELEMENT STEP SPECIFICATION

\begin{tabular}{|c|c|c|}
\hline Step & $\mathscr{F}\left(\mathrm{x}(/ \mathrm{m}), \mathrm{y}(/ \mathrm{m}), \theta\left(/{ }^{\circ}\right)\right.$ & Cost \\
\hline$\# 1$ & $(0.00,0.00,0)$ & 0.90 \\
\hline$\# 2$ & $(0.04,0.00,0)$ & 0.85 \\
\hline$\# 3$ & $(0.06,0.00,0)$ & 0.80 \\
\hline$\# 4$ & $(0.00,0.04,0)$ & 0.95 \\
\hline$\# 5$ & $(0.04,0.02,30)$ & 1.00 \\
\hline
\end{tabular}

Step \# 1 performs the functions of switching supporting foot during the searching process to realize higher planning efficiency.

As to Step \#5, the unique correlated HipYawPitch joint, the left foot could only turn counter-clock wise while the right foot could only turn clockwise. The element search cost is determined by displacement and the complexity of the movement.

The execution of the element step is conducted by sending the pre-calculated values for the 12 joints from the lower body the control period is $100 \mathrm{~ms}$.

\section{B. Ball Tracking Experiment}

In this experiment, the goal point, which is represented by a red ball, is moveable and the biped robot will track the red ball until the robot gets the ball. The experimental results are demonstrated in Fig. 9.

The robot was placed at the initial position and a red ball representing the goal was placed in the front of it. During the navigation, the simulator will carry out footstep re-planning by considering the present depth map and goal position. During the walking process, the robot will adapt to the change of goal position and adjust its footstep sequence to reaches goal.

\section{Reaction to Dynamic Experiment}

In this experiment, the environment was set dynamic and the robot would react to the uncertain environment while approaching the red ball. The experiment results are demonstrated in Fig. 10.

In this experiment, the goal position was set fixed while the obstacle was movable(represented by a blue box). During the navigation procedure, the obstacle was moved around to simulate the human-living environment. The robot will adjust its footstep sequence only when the obstacle lies in its routine.The movement of the obstacle is controlled by programmer to avoid the "local minima" situations which lead to searching failure.

\section{CONCLUSION AND FUTURE WORK}

This paper presented a framework for the simulation of biped navigation in human-living environment. $A^{*}$ based searching algorithm is utilized as the footstep planner. An offline sampling-based element step library is built as the database for the footstep planner, and a 3D Depth map is generated to provide the environment information for the planner. Webots platform and a NAO robot are adopted to build the experiment scene. Two specific experiments considering the change of the goal position and the environment were conducted to validate the proposed framework.

Physical experiments have been conducted and the physical performance varies a lot from the simulated one: because of the poor rigidity of the plastic, the main material of the NAO robot, external angle compensations are required for the HipRoll joints and AnkleRoll joints. Due to the uncertainty of the friction of the floor material, the actual displacement of the robot's feet is not accurate which would greatly reduce 


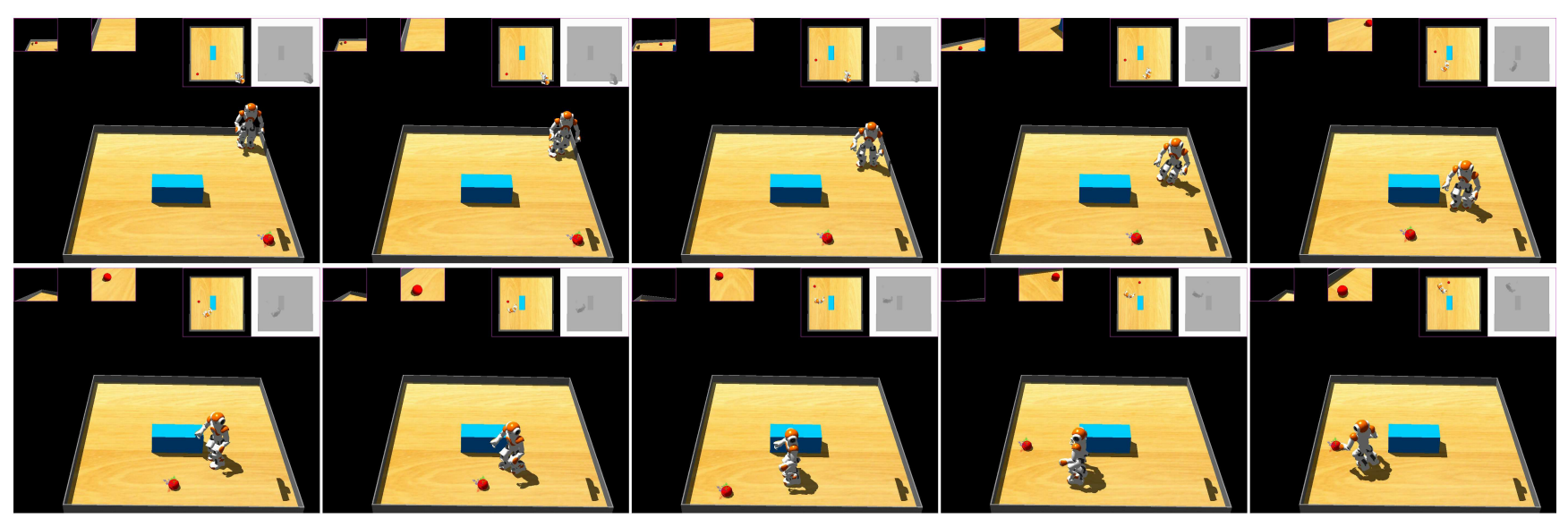

Fig. 9. Ball tracking experiment.

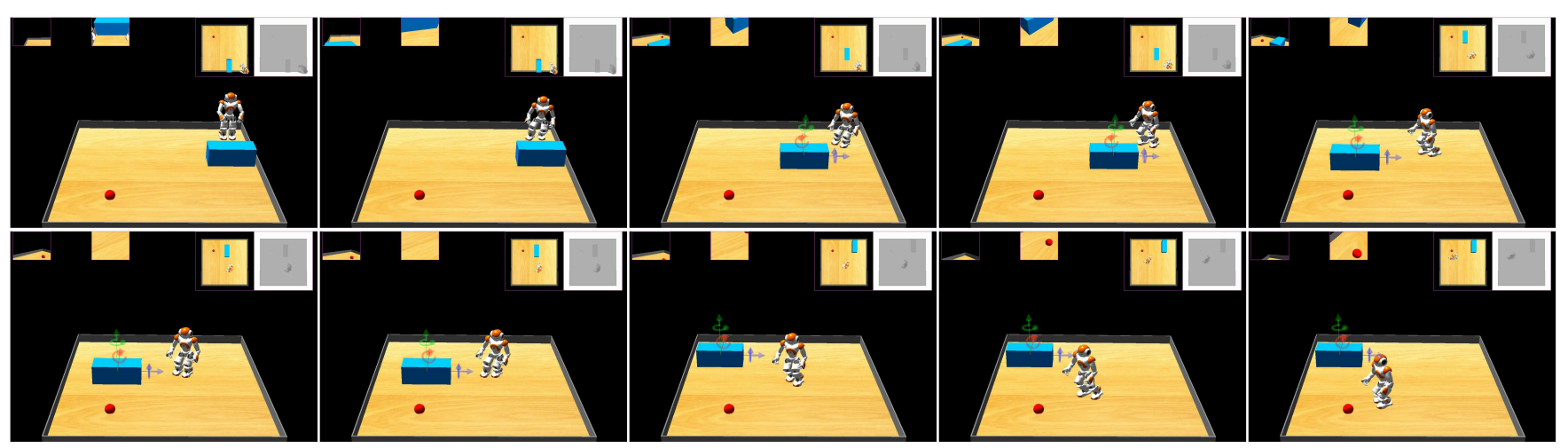

Fig. 10. Reaction to dynamic environment.

the advantage of the sampling based biped navigation. In the future, external sensors would be added to two feet and the torso part to get the actual posture of the robot and a closed loop control method would be used to control the execution of the element step by real-time sensing and angle compensation.

\section{REFERENCES}

[1] J. C. Latombe, Robot Motion Planning. Boston, MA: Kluwer, 1991

[2] J. J. Kuffner, K. Nishiwaki, K. Kgami, M. Inaba, and H. Inoue, Footstep Planning among obstacles for biped robots, in Proc. IEEE/RSJ Int. Conf. Intell. Robots Syst., Maui, Hawaii, Oct.2001, pp.500-505

[3] J. J. Kuffner, K. Nishiwaki, K. Kgami, M. Inaba, and H. Inoue, Motion planning for humanoid robots, Trans. Adv. Robot., vol.15, pp.365-374, 2005

[4] J. Chestnutt, J. Kuffner, K. Nishiwaki, and S.Kagami, Planning biped navigation strategies in complex environments, presented at the IEEE Int. Conf. Humanoid Robot., Munich Germany, 2003

[5] J. Chestnutt, M.Lau, J. Kuffener, J. Hodgins, and T.Kanada, Footstep planning for the Hongda ASIMO humanoid, in Proc. IEEE Int. Conf. Robot. Autom, Tsukuba, Japan, 2005, pp. 629-634

[6] J. Chestnutt, K. Nishiwaka, J. Kuffner, and S. Kagami, "An adaptive action model for legged navigation planning, in Proc. IEEE/RAS Int. Conf. Humanoid Robots, 2007, pp.196-202

[7] Z. Xia, J. Xiong and K. Chen, Parameter self-adaptation in biped navigation employing non-uniform randomized footstep planner, Robotica 28, 2010, pp. 361-366

[8] Z.Xia J.Xiong and K. Ken, Global Navigation for humanoid robots using sampling-based footstep planner, IEEE/ASME Transactions on Mechtronics vol 16, 2011, pp. 716-723
[9] Z. Xia, J. Xiong, and K. Chen, A deterministic sampling-based approach to global footstep planning for humanoid robots, in Proc. 2009 IEEE Int. Conf. Humanoid Robots (Humanoids), Paris, France, Dec. 7-10, 2009, pp.142-147

[10] R. Pfeifer, M. Lungarella, and F. Iida, Self-organization, embodiment, and biologically inspired robotics, Science, vol. 318, pp. 1088-1093, 2007

[11] Ijspeert, A.J., Central pattern generator for locomotion control in animals and robots: A review, Neural Networks, vol. 21, pp.642-653, 2008

[12] O. Michel, Cyberbotics Ltd, WebtosTM: Professional mobile robot simulation, International Journal od Adanced Robotic System vol1, 2004, pp. 39-42

[13] Microsoft Kinect http://www.microsoft.com/en-us/kinectforwindows/ 